\title{
Outbreak Investigations: Red-Eyed Rabbits and Community Service
}

\author{
Clare F. Pegues, RN, MPH
}

In the fall of 1984, the Department of Epidemiology and Public Health of the Yale University School of Medicine was awarded a Centers for Disease Control and Prevention demonstration grant to develop a program that would tackle statewide health problems and disease outbreaks by improving the cooperation between the school and the Connecticut Department of Health Services. Faculty from the school, in collaboration with Dr. James L. Hadler, MD, MPH, the State Epidemiologist, supervised small groups of second-year Masters in Public Health students to respond to statewide public health problems and outbreaks. The training included didactic lectures and a field investigation. As a member of one of the first groups, in the fall of 1985 , it was a tremendous experience for me as a public health student to learn to develop a systematic approach to outbreak investigations. ${ }^{1}$

This issue of the journal has several articles on outbreak investigations. Healthcare epidemiologists investigate disease outbreaks for several reasons. The primary goal is to identify the exposure or source to prevent further morbidity and mortality. ${ }^{2}$ To do this, we identify risk factors for the exposure and provide recommendations to clinicians and the public to prevent future outbreaks. In so doing, we are providing a community service for health promotion and disease prevention. In outbreak investigations, the goal is to rapidly identify the source(s) of the outbreak and implement control measures. Pressure from the individuals or organizations involved to quickly identify the source or etiologic agent can lead to premature and sometimes erroneous conclusions. The reputation of a restaurant or hospital involved in an outbreak is at stake, and their daily financial losses can be significant.

This issue of the journal includes 3 reports on eye infections, including 2 outbreaks of endophthalmitis. The incidence of acute-onset endophthalmitis after cataract surgery is approximately $0.05 \%$, depending on the surgical technique and perioperative eye preparations that are used..$^{3-5}$ Because of the low incidence of endophthalmitis, risk factors have been difficult to identify. West et al. ${ }^{6}$ identified age of more than 90 years and black race as possible risk factors. Miller et al. ${ }^{5}$ identified intraoperative complications, relative immunosuppression, the use of lidocaine $2 \%$ gel before povidone-iodine application, and inferior incision location as risk factors. Outbreaks of en- dophthalmitis have been associated with intrinsic contamination of ophthalmic solutions, ${ }^{7}$ contaminated instruments or equipment,${ }^{8,9}$ contaminated intraocular lens, ${ }^{10}$ and perioperative eye preparation. ${ }^{11}$ In addition, airborne exposure in the operating room has been reported, including a cluster of cases of Acremonium kiliense endophthalmitis associated with a contaminated ventilation system, ${ }^{12}$ and ocular aspergillosis associated with hospital construction. ${ }^{13}$

Acute-onset bacterial endophthalmitis is an ophthalmologic emergency that can cause permanent loss of vision if not diagnosed and treated promptly. The investigations of outbreaks of eye infection reported in this issue by AlonsoEchanove et al., ${ }^{14}$ Hugonnet et al., ${ }^{15}$ and Mateos et al. ${ }^{16}$ illustrate how a careful, methodical approach resulted in control of the outbreaks, advanced our knowledge of perioperative infection control in eye surgery, and addressed the potential utility of leukocyte-reduction filters for red blood cell transfusions.

In the outbreaks studied by Hugonnet et al. ${ }^{15}$ and Mateos et al., ${ }^{16}$ there were breaches of standard infection control procedures, including inadequate sterilization of equipment, extrinsic contamination of solutions, and failure to adhere to established surgical operative and postoperative standards. In the study by Alonso-Echanove et al., ${ }^{14}$ use of filters that were designed to minimize systemic illness instead caused local eye reactions.

Alonso-Echanove and colleagues conducted an elegant investigation of a nationwide outbreak of red eye syndrome associated with receipt of red blood cell transfusions filtered through leukocyte-reduction filters (LeukoNet; HemaSure). ${ }^{14}$ The filters were used to reduce the incidence of systemic, febrile, transfusion-associated adverse reactions. The authors conducted a nationwide survey, performed case-control and cohort studies, and reproduced red eye reactions in 17 unfortunate New Zealand white rabbits. Their meticulous work paid off; they showed a significant association between red eye syndrome and receipt of LeukoNet-filtered red blood cell transfusions, as well as a clear dose-response relationship, and the outbreak promptly ceased when the filters were pulled from the market.

Hugonnet et al. ${ }^{15}$ conducted an investigation of an outbreak 
of post-cataract extraction endophthalmitis associated with a particular surgeon in a university teaching hospital in Geneva, Switzerland. They focused their case-control study around the surgeon, who had operated on all of the case patients. They examined the operative procedure, surgical techniques, instruments, and sterilization procedures. By careful examination of instruments and comparison of the surgeon's technique with that of other hospital eye surgeons, the authors identified several risk factors for infection: use of several different surgical instruments (including a diamond blade), failure to properly suture the corneal tunnel, and failure to apply topical antibiotics after surgery-all issues pertaining to the surgical incision. In addition, the instrument sterilization was suboptimal and resulted in damage to the diamond blade. After the surgeon agreed to use disposable blades, suture the incision and apply topical antibiotics, the outbreak ended.

The investigation by Mateos et al. ${ }^{16}$ of an outbreak of Pseudomonas aeruginosa post-cataract extraction endophthalmitis in a hospital in southern Spain rapidly focused on intraoperative fluids. The investigation pointed to exogenous contamination of hospital pharmacy-reconstituted trypan blue solution, which was used to stain the ocular cavity intraoperatively. In addition, the sterilized trypan blue solution was not filtered through a $0.22-\mu \mathrm{m}$ pore filter before use, and the autoclaving process used to sterilize the trypan blue solution before use was reportedly "defective," although the specific problem was not identified by Mateos et al. ${ }^{16}$ The outbreak ended when the trypan blue stock solution and contaminated vials were removed from use.

The concurrent performance of laboratory investigations can either confirm or dispel theories about possible etiologies of an outbreak. In the study by Alonso-Echanove et al., ${ }^{14}$ it was the red-eyed rabbits that made the investigation particularly impressive, because the investigators essentially reproduced the clinical findings and thus confirmed the suspected exposure in the laboratory, an uncommon feat in healthcare epidemiology. In the report by Hugonnet et al., ${ }^{15}$ culture of environmental samples and perioperative fluid samples did not yield the outbreak pathogen, which confirmed the authors' theory that the outbreak was not associated with operating room or perioperative fluid contamination. In the study by Mateos et al., ${ }^{16}$ patient isolates and samples of reconstituted trypan blue stock solution and prepared vials all grew the same strain of $P$. aeruginosa, (confirmed by repetitive-element polymerase chain reaction), suggesting that use of the contaminated solution resulted in ocular infections.

In each of these investigations, the authors explored a limited number of biologically plausible hypotheses to gain insight into the etiology of the outbreak. Alonso-Echanove et al. ${ }^{14}$ hypothesized that the cellulose-acetate derivatives in the leukocyte-reduction filters leached through the filter membranes, causing the ocular reactions. They also raised the possibility that incorrect storage of the filters may have contributed to the leaching. Hugonnet et al. ${ }^{15}$ hypothesized that the damaged diamond blade that was used incorrectly compromised the surgical wound at closure, resulting in "ragged edges" and probable entry of microorganisms. In the study by Mateos et al., ${ }^{16}$ culture of samples of the autoclave water used to sterilize the solution failed to yield the outbreak strain. However, a different strain of $P$. aeruginosa was isolated, as well as Stenotrophomonas maltophilia and Achromobacter $x y$ losoxidans. The authors postulated that the trypan blue solution may have become contaminated with bacteria in the autoclave water during the cooling phase.

When hypotheses can be tested in the laboratory, as in the investigation by Alonso-Echanove and colleagues, ${ }^{14}$ the findings can be compelling. Alonso-Echanove et al. ${ }^{14}$ demonstrated that the ocular findings could be reproduced in an animal model, lessening the potential that recall bias or misclassification bias contributed to the observed association between use of the leukocyte-reduction filter and red eye syndrome. Elegant laboratory confirmation is not always practical nor feasible. It is unlikely that Hugonnet and colleagues ${ }^{15}$ could have reproduced their "surgical wound" theory in an animal model without spending considerable time and expense and possibly raising the ire of local animal rights activists. Instead, they relied on the data extracted from a thorough interview and careful observation of the surgeon to identify multiple potential risk factors for the outbreak.

What are the implications of the results of these studies? Prevention of postoperative infection associated with ophthalmic surgery relies on scrupulous asepsis and careful surgical technique, but as the investigations of Hugonnet et al. ${ }^{15}$ and Mateos et al. ${ }^{16}$ demonstrate, problems with these procedures continue, resulting in substantial patient morbidity. The proper sterilization of surgical equipment and perioperative fluids is still a challenge. Will the American Red Cross recommend the standard use of LeukoNet filters in future red blood cell transfusions, trading a lower risk of systemic illness for a higher risk of local reactions? Alonso-Echanove et al. ${ }^{14}$ report that, as of $2005,65 \%-95 \%$ of all red blood cell units at the American Red Cross and American Blood Centers are voluntarily leukoreduced, apparently using the same brand of filter implicated in the outbreak (LeukoNet). This filter was placed on the market 6 months before the first case of red eye syndrome, was recalled during the outbreak, and presumably was reintroduced at a later point; no successor has been marketed since then. ${ }^{14}$ How can leukocyte-reduction filters (or perhaps their storage procedure) be improved so that they are safe and effective for patient use? Is there a better approach to asepsis and sterilization in the operating room? Millions of red blood cell units are transfused and thousands of cataract extractions are performed every year; Alonso-Echanove et al., ${ }^{14}$ Hugonnet et al., ${ }^{15}$ and Mateos et al. ${ }^{16}$ have given us the tools to provide a higher standard of patient care and have provided an important public health service. 
Address reprint requests to Clare F. Pegues, RN, MPH, 2700 Anchor Avenue, Los Angeles, CA 90064 (cfpegues@comcast.net).

\section{REFERENCES}

1. Helgerson SD, Jekel JF, Hadler JL. Training public health students to investigate disease outbreaks-examples of community service. Pub Health Rep 1988; 103:72-76.

2. Reingold AL. Outbreak investigations-a perspective. Emerg Infect Dis 1998; 4:2I-28.

3. Hanscom TA. Postoperative endophthalmitis. Clin Infect Dis 2004; 38: 542-546.

4. Eifrig CW, Flynn HW Jr, Scott IU, Newton J. Acute-onset postoperative endophthalmitis: review of incidence and visual outcomes (1995-2001). Opthalmic Surg Lasers 2002; 33:373-378.

5. Miller JJ, Scott IU, Flynn HW, Smiddy WE, Newton J, Miller D. Acuteonset endophthalmitis after cataract surgery (2000-2004): incidence, clinical settings, and visual acuity outcomes after treatment. Am J Ophthalmol 2005; 139:983-987.

6. West ES, Behrens A, McDonnell PJ, Tielsch JM, Schein OD. The incidence of endophthalmitis after cataract surgery among the US Medicare population increased between 1994 and 2001. Ophthalmology 2005; 112: 1388-1394.

7. Outbreaks of postoperative bacterial endophthalmitis caused by intrinsically contaminated ophthalmic solutions-Thailand, 1992, and Canada, 1993. MMWR Morb Mortal Wkly Rep 1996; 45:491-494.

8. Cruciani M, Malena M, Amalfitano G, Monti P, Bonomi L. Molecular epidemiology in a cluster of cases of postoperative Pseudomonas aeruginosa endophthalmitis. Clin Infect Dis 1998; 26:330-333.

9. Hoffmann KK, Weber DJ, Gergen MF, Rutala WA, Tate G. Pseudomonas aeruginosa-related postoperative endophthalmitis linked to a contaminated phacoemulsifier. Arch Ophthalmol 2002; 120:90-93.

10. Pettit TH, Olson RJ, Foos RY, Martin WJ. Fungal endophthalmitis following intraocular lens implantation: a surgical epidemic. Arch Ophthalmol 1980; 98:1025-1039.

11. Mandal K, Hildreth A, Farrow M, Allen D. Investigation into postoperative endophthalmitis and lessons learned. J Cataract Refract Surg 2004; 30:1960-1965.

12. Fridkin SK, Kremer FB, Bland LA, Padhye A, McNeil MM, Jarvis WR. Acremonium kiliense endophthalmitis that occurred after cataract extraction in an ambulatory surgical center and was traced to an environmental reservoir. Clin Infect Dis 1996; 22:222-227.

13. Tabbara KF, al Jabarti AL. Hospital construction-associated outbreak of ocular aspergillosis after cataract surgery. Ophthalmology 1998; 105: 522-526.

14. Alonso-Echanove J, Sippy BD, Chin AE, et al. Nationwide outbreak of red eye syndrome associated with transfusion of leukoreduced red blood cell units. Infect Control Hosp Epidemiol 2006; 27:1146-1152 (in this issue).

15. Hugonnet S, Dosso A, Dhoran S, et al. Outbreak of endophthalmitis after cataract surgery: the importance of the quality of the surgical wound. Infect Control Hosp Epidemiol 2006; 27:1246-1248 (in this issue).

16. Mateos I, Valencia R, Torres MJ, Cantos A, Conde M, Aznar J. Nosocomial outbreak of Pseudomonas aeruginosa endophthalmitis. Infect Control Hosp Epidemiol 2006; 27:1249-1251 (in this issue). 